\title{
Evidence for Genetic Similarity of Vegetative Compatibility Groupings in Sclero- tinia homoeocarpa
}

\author{
Seog Won Chang ${ }^{1 *}$, Young-Ki Jo' ${ }^{2}$ Taehyun Chang ${ }^{3}$ and Geunhwa Jung ${ }^{4 *}$ \\ ${ }^{1}$ Turfgrass Science Institute, Hanul Inc., Hapcheon 678-803, Korea \\ ${ }^{2}$ Department of Plant Pathology and Microbiology, Texas A\&M University, College Station, TX, USA \\ ${ }^{3}$ Department of Plant Resources and Environment, College of Ecology and Environmental Science, Kyungpook National \\ University, Sangju 742-711, Korea \\ ${ }^{4}$ Stockbridge School of Agriculture, University of Massachusetts, Amherst, MA, USA
}

(Received on August 11, 2014; Revised on September 22, 2014; Accepted on September 30, 2014)

Vegetative compatibility groups (VCGs) are determined for many fungi to test for the ability of fungal isolates to undergo heterokaryon formation. In several fungal plant pathogens, isolates belonging to a VCG have been shown to share significantly higher genetic similarity than those of different VCGs. In this study we sought to examine the relationship between VCG and genetic similarity of an important cool season turfgrass pathogen, Sclerotinia homoeocarpa. Twenty-two S. homoeocarpa isolates from the Midwest and Eastern US, which were previously characterized in several studies, were all evaluated for VCG using an improved nit mutant assay. These isolates were also genotyped using 19 microsatellites developed from partial genome sequence of $S$. homoeocarpa. Additionally, partial sequences of mitochondrial genes cytochrome oxidase II and mitochondrial small subunit (mtSSU) rRNA, and the atp6-rns intergenic spacer, were generated for isolates from each nit mutant VCG to determine if mitochondrial haplotypes differed among VCGs. Of the 22 isolates screened, 15 were amenable to the nit mutant VCG assay and were grouped into six VCGs. The 19 microsatellites gave 57 alleles for this set. Unweighted pair group methods with arithmetic mean (UPGMA) tree of binary microsatellite data were used to produce a dendrogram of the isolate genotypes based on microsatellite alleles, which showed high genetic similarity of nit mutant VCGs. Analysis of molecular variance

\footnotetext{
*Co-corresponding author.

S. W. Chang

Phone) +82-55-934-0145, Fax) +82-55-934-0148

E-mail) changsw802@hanmail.net

G. Jung

Phone) +1-413-545-2243, Fax) +1-413-577-0242

E-mail)jung@umass.edu
}

of microsatellite data demonstrates that the current nit mutant VCGs explain the microsatellite genotypic variation among isolates better than the previous nit mutant VCGs or the conventionally determined VCGs. Mitochondrial sequences were identical among all isolates, suggesting that this marker type may not be informative for US populations of $S$. homoeocarpa.

Keywords : nit mutants, Sclerotinia homoeocarpa, microsatellites, VCG

The ascomycete fungus Sclerotinia homoeocarpa (Order: Pezizales, Family: Sclerotiniaceae) causes dollar spot, one of the most common and destructive fungal diseases on intensively managed turfgrasses in North America (Smith et al., 1989; Subbarao et al., 1995) and Korea (Chang et al., 2011). It is most prevalent on golf courses, and turfgrass damage from dollar spot is most severe during the growing season from spring to fall (Chang et al., 2012 and 2013; Smiley, 2005). Warm and humid daytime conditions coupled with cool nights promote extended periods of leaf wetness, which is particularly conducive to disease development. Demands for high aesthetic quality and playability of golf courses require low mowing heights, which also increases the susceptibility of turfgrasses to dollar spot infection. Accordingly, more money is spent on controlling dollar spot on golf courses in the US than any other disease (Burpee, 1997; Viji et al., 2004).

The conidial or teleomorphic forms of $S$. homoeocarpa have not been observed in the field and have not been produced in the laboratory, with the exception of a few cases of in vitro induction of apothecial sterile spores using isolates from the United Kingdom (Bennett, 1937; Jackson, 1973). It is assumed that the fungus spreads locally by veg- 
etative mycelium, and long distance dissemination may occur by infected grass clippings via wind, water, machinery, or human traffic (Baldwin and Newell, 1992; Subbarao et al., 1995). During the vegetative phase, it has been demonstrated that hyphal fusion and heterokaryosis may occur between mycelia within the same compatibility group (Glass et al., 2004; Jo et al., 2008). Vegetative hyphal fusions are vital to genetic exchange, translocation of water and nutrients, and general homeostasis within or between fungus populations in the field (Glass et al., 2000).

Vegetative compatibility refers to the ability of the hyphae of two individual fungal isolates to fuse together and form viable heterokaryons (Leslie, 1993). In fungi lacking sexual stages, vegetative compatibility may serve as an important means of genetic exchange and generating new genetic diversity (Leslie, 1993). Isolates that are vegetatively compatible with each other are said to be members of the same vegetative compatibility group (VCG) (Joaquim and Rowe, 1991; Leslie, 1993). Conversely, vegetatively incompatible isolates are incapable of establishing stable heterokaryosis.

The vegetative compatibility assay has been used to measure population diversity of fungi and to enable appropriate identification and characterization of individual isolates. Vegetative compatibility assays have been used to determine the population structures of many plant-pathogenic fungi, including Fusarium spp. (Katan and Katan, 1988; Marlatt et al., 1996), Verticillium spp. (Joaquim and Rowe, 1991), and Colletotrichum spp. (Brooker et al., 1991; Cecilia De Lima Favaro et al., 2007). The conventional method of determining VCGs involves the detection of a darkly pigmented lytic area, or barrage zone, where mycelium from two incompatible isolates meet. According to the conventional method VCG determination, if the barrage zone is absent, isolates are said to be vegetatively compatible. Another type of VCG assay that has been widely used relies on the complementation between nitrate-nonutilizing (nit) mutants (Leslie, 1993). This assay proceeds as follows: mutants deficient in one of several steps of the nitrogen assimilation pathway are made for each isolate tested. A single mutant is chosen from each of two parent isolates to be tested against one another, with each mutant deficient for a different step in the nitrogen assimilation pathway. They each are inoculated onto opposite ends of a petri plate with an agar medium amended with nitrate as the sole nitrogen source. The nit mutant exhibits sparse growth on the nitrate medium, but when the mycelium of compatible mutants meets, they fuse and form distinct and prolifically growing heterokaryotic mycelium, since the mutations of the nitrogen assimilation pathway are complemented (Cor- rell et al., 1998; Joaquim and Rowe, 1991; Katan and Ka$\tan$, 1988). This method of determining VCGs is preferred because it demonstrates the ability of isolates to form heterokaryotic mycelium.

Jo et al. (2008) developed the nit mutant assay for S. homoeocarpa to clear up confusion arising from ambiguous VCG results using the conventional method of VCG determination (Jo et al., 2008; Powell and Vargas, 2001). This assay revealed four VCGs from a panel of isolates shown to represent eight groups by Chakraborty et al. (2006) using the conventional method of VCG determination of Powell and Vargas (2001). Over half of the isolates tested were assigned to one of two dominant nit mutant VCGs (Jo et al., 2008), and the authors report that some of the compatible complementation interactions resulted only in weak heterokaryon formation. In this current study, the nit mutant VCG assay for S. homoeocarpa was optimized and carried out with isolates previously used by Powell and Vargas (2001), Chakraborty et al. (2006), and Jo et al. (2008). The new assay requires using a fourth mutant type (nit2), a much more robust sampling of mutants, and a longer incubation time for paired mutants than was previously performed. Our improved nit mutant assay gave slightly discordant results to the former nit mutant VCG assay of Jo et al. and showed that there were two more VCGs than had been described previously with the initial nit mutant VCG assay (Jo et al., 2008).

The objective of this work was to determine if the VCGs found with the improved nit mutant assay for S. homoeocarpa share significantly more genetic similarity of microsatellite and mitochondrial genotypes groupings, as compared to previous studies using these isolates (Chakraborty et al., 2006; Jo et al., 2008; Powell and Vargas, 2001).

\section{Materials and Methods}

Isolation of nit mutants. Twenty-two isolates of S. homoeocarpa used in this study originated from various geographical locations in the US including Arkansas, Illinois, Michigan, Florida, and Minnesota (Table 1). Powell and Vargas and Chakraborty et al. used these isolates previously for barrage based vegetative compatibility assays (Chakraborty et al., 2006; Powell and Vargas, 2001). The conventional VCGs of a majority of these isolates (VCGs A-F) were presented by Powell and Vargas (2001), and the remainder of the conventional VCG designations (VCGs G, H, J, K, and L) was made by Powell and Vargas (2001) for Chakraborty et al. (2006). Jo et al. (2008) used this entire panel of isolates for nit-based VCG (Jo et al., 2008). In the time between the experiments of Chakraborty et al. (2006), 
Table 1. Sclerotinia homoeocarpa isolates used, and their vegetative compatibility groups (VCGs) determined by pairings of nitrate nonutilizing (nit) mutants in this study and comparison with two previous studies

\begin{tabular}{|c|c|c|c|c|}
\hline Isolate & Site of collection & $\begin{array}{l}\text { Chakraborty et al. } \\
\qquad(2006)^{\mathrm{a}}\end{array}$ & $\begin{array}{l}\text { Jo et al. } \\
(2008)^{b}\end{array}$ & This study \\
\hline FL17 & Unknown golf course, FL & A & Group 1 & VCG 1 \\
\hline L36 & Unknown golf course, IL & A & Group 1 & VCG 1 \\
\hline MN1 & Unknown golf course, $\mathrm{MN}$ & A & Group 1 & VCG 1 \\
\hline $33 \mathrm{~A}-24$ & Hancock Turf Research Center, East Lansing, MI & $\mathrm{B}$ & - & VCG 1 \\
\hline ARK & Unknown golf course, AR & $\mathrm{B}$ & Group 1 & - \\
\hline Southbrook & Southbrook, MN & $\mathrm{B}$ & Group 1 & VCG 2 \\
\hline A7 & Southbrook, MN & $\mathrm{H}$ & Group 1 & - \\
\hline TB64D & Unknown & $\mathrm{K}$ & Group 1 & VCG 2 \\
\hline 30B-13 & Lakewood Shores, MI & $\mathrm{C}$ & Group 1 & VCG 2 \\
\hline 30B-24 & Lakewood Shores, MI & $\mathrm{C}$ & Group 1 & - \\
\hline $46-3$ & Forest Akers Golf, East Lansing, MI & $\mathrm{F}$ & Group 1 & VCG 2 \\
\hline 30B-48 & Lakewood Shores, MI & $\mathrm{D}$ & Group 1 & VCG 3 \\
\hline $48-54$ & North Shore County Club, Chicago, IL & $\mathrm{D}$ & Group 1 & - \\
\hline $64-41$ & Evergreen Golf, Hudson, MI & $\mathrm{E}$ & Group 1 & VCG 4 \\
\hline $64-49$ & Evergreen Golf, Hudson, MI & $\mathrm{E}$ & Group 1 & VCG 4 \\
\hline I16 & Les Bolstead, St. Paul, MN & G & Group 2 & VCG 5 \\
\hline I18 & Les Bolstead, St. Paul, MN & G & Group 2 & VCG 5 \\
\hline Les Bolstead & Les Bolstead, St. Paul, MN & $\mathrm{J}$ & Group 2 & VCG 5 \\
\hline BRS & Unknown & $\mathrm{L}$ & Group 2 & - \\
\hline $32-47$ & Evergreen Golf, Hudson, MI & $\mathrm{F}$ & Group 3 & VCG 6 \\
\hline $33 \mathrm{~A}-9$ & Hancock Turf Research Center, East Lansing, MI & $\mathrm{C}$ & Group 4 & - \\
\hline $64-14$ & Evergreen Golf, Hudson, MI & E & - & - \\
\hline
\end{tabular}

${ }^{a}$ Vegetative compatibility groups (VCGs) determined by barrage formation at pairings of wild type isolates on potato dextrose agar medium using the method of Powell and Vargas (2001).

${ }^{b}$ VCGs were determined using nit mutants but specific names were not assigned.

Jo et al. (2008), and this study, all isolates were stored in long term cultures of Kentucky bluegrass seed and potato dextrose broth at $4^{\circ} \mathrm{C}$.

The nit mutants of each $S$. homoeocarpa isolate were isolated using the modified procedure developed by Jo et al. (2008). At first, one 5-mm diameter plug was taken from the colony edge of each $S$. homoeocarpa isolate grown on potato dextrose agar (PDA), and transferred to $10 \mathrm{ml}$ potato dextrose broth (PDB) in a 9-cm Petri dish. After incubation for 10 days at $25^{\circ} \mathrm{C}$, mycelia were harvested and dried on sterilized paper towels. A $5 \mathrm{~g}$ sample of the mycelia was homogenized with $5 \mathrm{ml}$ sterile, distilled water using a blender (Brinkmann Instruments Co, Switzerland) at $15,000 \mathrm{rpm}$ for $30 \mathrm{~s}$. A $200 \mu \mathrm{l}$ aliquot of shredded mycelial suspension was spread on ten plates of water agar medium amended with chlorate (WAC: $2 \%$ agar, $0.2 \%$ glucose and $4 \%$ potassium chlorate) for screening the nit mutants. After spreading, all plates were air dried in a laminar flow hood for $5 \mathrm{~min}$ and incubated at $25^{\circ} \mathrm{C}$. When colonies of nit mu- tants became distinct, showing an expansive and thin mycelial growth on WAC after 30 days incubation, the hyphal tip of each colony was transferred to Czapek solution agar medium (CDA; Becton, Dickson and Company, Sparks, $\mathrm{MD}$ ) containing nitrate as a single source of nitrogen. Each colony transferred was examined for the typical nit mutant phenotype: a thin and expansive growth with no aerial mycelium on CDA (Correll et al., 1987). Confirmed nit mutants were sub-cultured on WAC and stored at $4^{\circ} \mathrm{C}$. The screening of nit mutants from the 22 isolates was repeated twice, independently.

Colony-forming units (CFUs) from the shredded mycelial suspension were measured to estimate the mutation rate. Serial dilutions of mycelial suspension of each isolate (wild type) were made at $10^{-1}, 10^{-2}$ and $10^{-3}$ using sterile, distilled water. A $20 \mu 1$ aliquot of the shredded mycelial suspension was spread on four PDA plates. After incubation at $25^{\circ} \mathrm{C}$ for 3 days, colonies were counted. The frequency of nit mutants was determined as the number of isolated nit 
Table 2. Identification of four types of nitrate nonutilizing (nit) mutants in Sclerotinia homoeocarpa grown in Czapek solution agar medium amended with different nitrogen sources

\begin{tabular}{|c|c|c|c|c|}
\hline \multirow{2}{*}{ Function ${ }^{\mathrm{a}}$} & \multirow{2}{*}{$\begin{array}{c}\text { Mutant } \\
\text { designation }\end{array}$} & \multicolumn{2}{|c|}{ Growth on nitrogen sources ${ }^{b}$} & \multirow{2}{*}{$\begin{array}{c}\text { Nitrite } \\
\text { excretion }\end{array}$} \\
\hline & & Nitrite & Hypoxanthine & \\
\hline Structural gene for nitrate reductase & nit 1 & + & + & NT \\
\hline Major nitrogen regulatory gene & nit2 & - & - & NT \\
\hline Pathway-specific regulatory gene & nit3 & - & + & - \\
\hline Genes controlling production of a molybdenum-containing cofactor & NitM & + & - & NT \\
\hline None & Wild type & + & + & + \\
\hline
\end{tabular}

${ }^{a}$ Compiled from Correll et al. (1987), Marzluf (1981), and Tomsett and Garrett (1980) on the basis of mutant phenotypes used for Fusarium oxysporum, Aspergillus nidulans, and Neurospora crassa, respectively.

${ }^{\mathrm{b}}$ Growth on Czapek solution agar (CDA) medium amended with two nitrogen sources. $+=$ typical wild-type growth and $-=$ thin growth with no aerial mycelium.

'Nitrite excretion test as described by Cove (1976). $+=$ nitrite excretion, $-=$ no nitrite excretion, and NT $=$ not tested.

mutants divided by the total number of CFUs on PDA per the same amount of mycelial suspension.

Characterization of nit mutant phenotypes. Specific phenotypes of nit mutants were determined by their utilization of two different nitrogen substances: $\mathrm{NaNO}_{2}$ and hypoxanthine (both obtained from Sigma-Aldrich) (Correll et al., 1987). Agar plugs (5 mm in diameter) were taken from the edge of each nit mutant culture growing on CDA, and transferred to three types of media: CDA, CDA amended with $\mathrm{NaNO}_{2}(0.05 \% \mathrm{w} / \mathrm{v})$, and CDA amended with hypoxanthine $(0.02 \% \mathrm{w} / \mathrm{v})$. An agar plug of each original wildtype isolate was also transferred to $\mathrm{CDA}$ as a wild-type control for each test run. The plates were incubated at $25^{\circ} \mathrm{C}$ for 10 days and the colony morphology was examined. Nit mutant phenotypes were determined by following the previously published nomenclature system (Correll et al., 1987; Marzluf, 1981). Mutants were divided into four phenotypic classes which are associated with mutated loci encoding members of the nitrogen assimilation pathway. These nit mutants were classified based on mutation of the following loci: a nitrate reductase structural locus (nit 1 ), a major nitrogen regulatory locus (nit2), a pathway-specific regulatory locus (nit3), and the locus encoding the cofactor containing molybdenum necessary for nitrate reductase activity (NitM). Mutants unable to use nitrate but able to utilize both nitrite and hypoxanthine were designated as nit1. Mutants incapable of using nitrate, nitrite and hypoxanthine were referred to as nit2. Mutants that could not use nitrate and nitrite but could use hypoxanthine were designated as nit3. Mutants capable of utilizing nitrite but not nitrate and hypoxanthine were designated as NitM (Table 2).

Subsequently, all nit 3 mutants were further tested for nitrite excretion using the procedures by Correll et al.
(1987). Each nit3 isolate was grown on urea medium (CDA amended with $0.04 \%$ urea) in a $9-\mathrm{cm}$ Petri dish at $25^{\circ} \mathrm{C}$ for 3 days. The plate was then flooded with $10 \mathrm{ml}$ of $3 \mathrm{M}$ $\mathrm{NaNO}_{3}$ solution (Sigma-Aldrich). After $24 \mathrm{~h}$ incubation at $25^{\circ} \mathrm{C}, \mathrm{NaNO}_{3}$ was poured off. By adding $1 \mathrm{ml}$ of a sulfanilamide solution and $1 \mathrm{ml}$ of a color indicator to the plate, the presence of nitrite was indicated by distinct purple color reaction.

Vegetative compatibility assay. Different types of nit mutants selected from each of the isolates tested were paired in all possible combinations. Agar plugs (5 $\mathrm{mm}$ in diameter) were cut from the edge of each nit mutant colony actively growing on CDA. Agar plugs from two different isolates were placed $4 \mathrm{~cm}$ apart on a $9-\mathrm{cm}$ diameter plate of CDA (Fig. 1). The plates were incubated at $25^{\circ} \mathrm{C}$ and monitored for 30 days. A complementary reaction was evident by the development of dense aerial mycelial growth. The pairing experiment was conducted twice.

Genome sequencing and development of microsatellite markers. Genomic sequences were generated using 454 Next Generation Sequencing and sequencing to up to $8 \mathrm{X}$ depth of the S. homoeocarpa isolate SD-20 from Joseph Troll Turfgrass Research Center, South Deerfield, MA (Macrogen Inc.). The assembled data represented over 34,000 contigs, each over $100 \mathrm{bp}$ in size and totaling over $30 \mathrm{Mb}$ of genome sequence. The contigs generated were searched for microsatellites using the Simple Sequence Repeat Identification Tool (SSRIT) (http://www.gramene. org/db/markers/ssrtool). Search parameters specified octamer as the maximum motif length and the minimum number of repeats at ten. Three-hundred nine SSRs were identified, and 96 of these were selected for further 


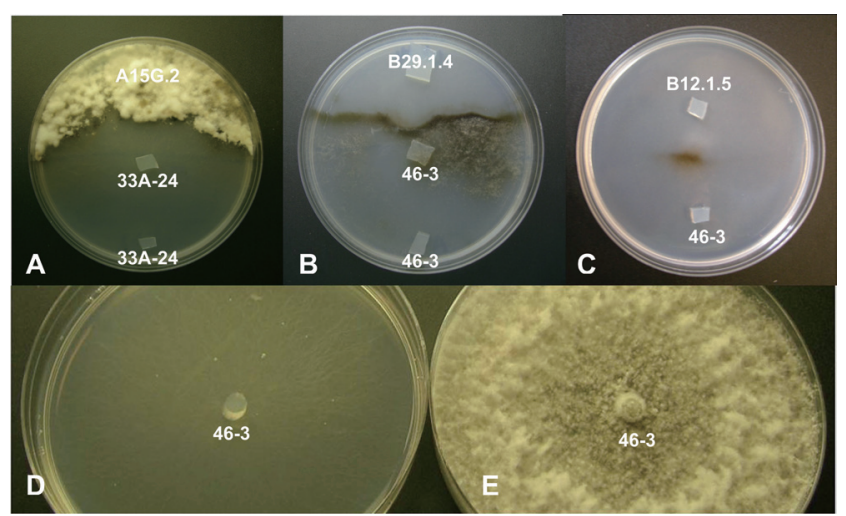

Fig. 1. Vegetative compatibility reactions between nit mutants of Sclerotinia homoeocarpa on Czapek solution agar medium (CDA) after 30-day incubation at $25^{\circ} \mathrm{C}$. A, Formation of vigorous heterokaryotic mycelium at the contact site between compatible isolates (A15G.2 and 33A-24). B. Formation of a distinct barrage with dark pigmentation between incompatible isolates (B29.1.4 and 46-3). C. Formation of small spot of aerial mycelium 2 weeks after incubation but later a barrage with dark pigmentation between incompatible isolates (B12.1.5 and 46-3). D. Mycelia of isolate 46-3 grown on CDA after 30-day incubation at $25^{\circ} \mathrm{C}$. E. Mycelia of isolate 46-3 grown on potato dextrose agar medium (PDA) after 7-day incubation at $25^{\circ} \mathrm{C}$. screening.

Forward and reverse PCR primers flanking the repeat sequences were designed using the Primer3 primer design server (Rozen and Skaletsky, 2000). The parameter settings used to generate primer sequences were as follows: primer length between 18 and 25 bases and annealing temperature from 55 to $65^{\circ} \mathrm{C}$. Primers were designed to amplify products ranging from 100 to 250 bases.

Microsatellites were amplified in a $10 \mu \mathrm{l}$ reaction volume with a final concentration of $1 \times$ PCR Buffer, 0.2 $\mathrm{mM}$ dNTP with $2.5 \mathrm{nM} \mathrm{MgCl}, 0.2 \mu \mathrm{M}$ of each primer, 1 unit Taq DNA Polymerase (New England Biolabs Inc.) and $50 \mathrm{ng}$ of genomic DNA template. The DNA for PCR was prepared using the method of Saitoh et al. (2006). The PCR regime was as follows: an initial denaturation at $95^{\circ} \mathrm{C}$ for $60 \mathrm{~s}$, followed by 35 cycles of $95^{\circ} \mathrm{C}$ for $60 \mathrm{~s}$, annealing at $72^{\circ} \mathrm{C}$ for $1 \mathrm{~min}$ and decreasing by increments of 1.0 degree per cycle for the first 10 cycles, and $72^{\circ} \mathrm{C}$ for $90 \mathrm{~s}$, with a final extension of $72^{\circ} \mathrm{C}$ for $5 \mathrm{~min}$. The PCR reactions were performed in 96 well plates on an MJ PTC200 thermocycler (MJ Research), with one reaction per primer pair without template as a negative control. Eight microliters of the PCR products and $2 \mu$ of $6 \times$ loading dye

Table 3. Descriptions of nineteen microsatellite loci mined from the S. homoeocarpa genome sequence using SSR-IT and the forward and reverse primer sequences used to amplify these markers. Primer sequences are listed in 5' to 3' orientation. The number of alleles among the 22 isolates genotyped is listed

\begin{tabular}{|c|c|c|c|c|c|}
\hline Sequence & Repeat motif $^{a}$ & $\begin{array}{l}\text { Size } \\
\text { (bp) }\end{array}$ & Left primer & Right primer & No. alleles \\
\hline SSR02063 & $(\mathrm{CTCAC})_{12}$ & 243 & CCTTGGCAGCCTCTGATTAT & TGAGGGTTCATGGAATAGCA & 2 \\
\hline SSR05848 & $(\text { GTATGA })_{12}$ & 232 & TTGGTGTAGGTGGAGGCTAGA & CTCACGTTCACTCACGCACT & 2 \\
\hline SSR06235 & $(\mathrm{TG})_{17}$ & 197 & TTCTTCCTTTCGGGTGACAG & CTTTTTCGTCTGCCTTGTGG & 3 \\
\hline SSR08045 & $(\mathrm{AC})_{13}$ & 157 & GTCGTGGAGAGGAGAGGTGA & AACGCGAGCCAACACTATCT & 4 \\
\hline SSR08400 & $(\mathrm{ATCT})_{12}$ & 202 & CTATTCTCGCGCATCCTCAT & TCGACGGTATCCTAGCAAGTG & 3 \\
\hline SSR08569 & $(\text { TATGGGA })_{13}$ & 227 & CCTCTCGTTCCTGGTTTCAC & CAATATCCATCCATCCATCCA & 3 \\
\hline SSR09987 & $(\mathrm{CA})_{12}$ & 233 & CTCCAACCAATCСТCСТTGA & TGGGCTACCGAGTACTTTGC & 2 \\
\hline SSR15983 & $(\mathrm{TAG})_{13}$ & 150 & TATAGCTCGCGGATGATGTG & AGACGGACTTACGCAATGCT & 4 \\
\hline SSR17616 & $(\mathrm{AC})_{16}$ & 172 & TCCGCACTACCGTTACACAC & GTGCGATGGAGATGGAGTCT & 2 \\
\hline SSR20589 & $(\mathrm{TCA})_{13}$ & 243 & ATCGACCCAAGAATCACCAA & AGGCTGGGTGCCTTAGTTTT & 2 \\
\hline SSR20624 & $(\mathrm{TTCA})_{16}$ & 184 & AGTTGGGCGAACGAATAAGA & GGCTGAAAGGGAGAAAGAACA & 3 \\
\hline SSR21791 & $(\mathrm{GAA})_{12}$ & 215 & CCATTCGTTCTATGGGTTCG & GGGACTTCTCCTTCCCATTC & 2 \\
\hline SSR22804 & $(\mathrm{AT})_{15}$ & 232 & CGGCTAGTTCGTCAATCAGG & AGCGGACGAGGAGGTAAACT & 2 \\
\hline SSR23039 & $(\mathrm{TC})_{16}$ & 197 & TCTTGCTCTGCTCTGCTCTG & TGGCCTTTTGCTTGCTTACT & 3 \\
\hline SSR25827 & $(\mathrm{AATC})_{15}$ & 188 & ССТTCСТTTCCAGCCTATCC & CCCGCTTTTTGGTTTTTGT & 5 \\
\hline SSR27998 & $(\text { GTTAT })_{12}$ & 165 & ATTGATGGGCATCGGTTG & TCСТСТССТСТССТССТСТGTA & 4 \\
\hline SSR30326 & $(\text { TTGAC })_{14}$ & 167 & GCAATGAGTGAGCGTCTTGT & TCATATCATCAAACGCATCCA & 4 \\
\hline SSR30530 & $(\mathrm{TC})_{12}$ & 156 & AATCGAGCACAGTCCAGTCC & TCTGTCTACTTGTCCGTCGATTT & 3 \\
\hline SSR30647 & $(\mathrm{AC})_{14}$ & 170 & GCTGTGGCCCATAATACGAT & GGCTGGATGTGCTGGATAAA & 2 \\
\hline
\end{tabular}

${ }^{\mathrm{a}}$ Repeat motif determined from genome of $S$. homoeocarpa isolate SD-20. 
per reaction were mixed and amplicons separated by gel electrophoresis on 3\% (w/v) MetaPhor agarose (Cambrex Bio Science Rockland, Inc.) gels in $1 \times$ TBE buffer (Tris base, Boric acid, and EDTA) at $160 \mathrm{~V}$ for 2.5 hours. Gels were post-stained with ethidium bromide $(0.5 \mu \mathrm{l} / \mathrm{ml})$ for 30 minutes, followed by 10 minutes destaining in $1 \mathrm{X}$ TBE buffer. Gel bands were visualized under UV light, photographed and gel pictures were captured as TIFF files for further analysis.

Microsatellites were screened initially for polymorphism among a panel of four isolates (FL17, ARK, I18, and 33A9) (Table 1). Microsatellite primer pairs were selected for further analysis if they were found to amplify single bands and were found polymorphic among the panel of four isolates. Nineteen microsatellite primer pairs were chosen based on this initial screening, and these primers were utilized to genotype the 22 isolates from Table 1 (Table 3 ).

Amplification and sequencing of mitochondrial loci. Primers were designed from partial genome sequence of $S$. homoeocarpa. Two mitochondrial loci, the mitochondrial small subunit rRNA (mtSSU) and the atp6-rns intergenic spacer region of the mitochondrial genome, were chosen for sequencing based on their utility as intraspecific genetic markers in other studies of pathogenic ascomycete fungi (Ghikas et al., 2010; Skovgaard et al., 2001). The partial gene sequence of cytochrome oxidase II (COII) was examined to look for variation, as well, though there are no reports of this gene showing intraspecific variability for ascomycete fungi. These genes were mined from $S$. homoeocarpa genomic scaffolds using a local BLAST search (Altschul et al., 1997). Primers for amplification were designed in Primer3 (Rozen and Skaletsky, 2000) to genomic scaffolds using the following criteria: minimum primer length 20 , optimum primer length 24 , maximum primer length 27 ; minimum melting temperature $67^{\circ} \mathrm{C}$, optimum melting temperature $70^{\circ} \mathrm{C}$, and maximum melting temperature $73^{\circ} \mathrm{C}$. The forward and reverse primer sequences for $\mathrm{mtSSU}$ were 5'-GCTGAACCAGCAACTTGGGGGAAT-3' and 5'-TCCCTTCCACGAATTCACACTCCA-3', and amplified an $838 \mathrm{bp}$ amplicon. The forward and reverse primer sequences for the atp6-rns spacer were 5'-GCAGAGGACTTTCTCGTGCAAAGCA-3' and 5'-GGGTAGGGGAGACAAACCTCCCACTT-3', and amplified a $721 \mathrm{bp}$ amplicon. The forward and reverse primer sequences for the COII locus were 5'-GTCGCCTGGCGGAGGGAGTA-3' and 5'-GCGGAGCTAGAGCTTGCTGTGTC-3', and amplified a 1091 bp amplicon. The PCR temperature regime followed that of Lamour et al.
(2006).

Microsatellite data analysis. Microsatellite alleles were coded into a binary data matrix, and this dataset was converted into a genetic distance matrix using the program GeneAlEx, and add in for population genetics analyses in Microsoft Excel (Peakall and Smouse, 2006). The genetic distance matrix was exported and analyzed in the program MEGA v4.0 using UPGMA distance analysis (Tamura et al., 2007). Additionally, the binary data matrix was interrogated with 10,000 bootstrap replicates using Phylip genetic analysis software package (Felsentein, 1989). The bootstrapped data matrices were converted to distance matrices, and a consensus tree was obtained.

The microsatellite distance matrices were also analyzed to determine genetic distance among and within VCGs found here, those of Jo et al. (2008), those of Chakraborty et al. (2006), which were determined with the conventional method of Powell and Vargas (2001). The isolates for which nit mutant VCG was determined in the current study and the previous two studies were analyzed for genetic relationships among and between the VCGs with more than one isolate (Chakraborty et al., 2006; Jo et al., 2008; Powell and Vargas, 2001).

Analysis of molecular variance (AMOVA) of microsatellite genotypes and VCGs. Binary matrices of microsatellite allelic data from three data sets were analyzed using GeneAlEx v6.4 for an Analysis of Molecular Variance (AMOVA). An AMOVA was used to determine how much of the microsatellite genetic variation could be explained by the VCG groupings of this current study and previous studies (Jo et al., 2008; Powell and Vargas, 2001). AMOVA was performed for isolates from VCGs with more than one isolate as is required for AMOVA, and only included isolates which were genotyped in this study. Three separate AMOVAs were carried out to determine which VCG designations explained the microsatellite genotypic variation best: our nit mutant VCGs, those of Jo et al. (2008), or those of Chakraborty et al. (2006). For each analysis, 9,999 pairwise permutations and total permutations were carried out with calculated distances based on haploid-SSR parameters.

\section{Results}

Isolation of nit mutants. A total of 101 nit mutants were isolated from 16 out of $22 \mathrm{~S}$. homoeocarpa isolates tested in this current study, and six isolates (A7, ARK, BRS, 30B$24,48-54$ and 64-14) failed to produce any nit mutants in 
Table 4. Frequency and phenotype of nitrate nonutilizing (nit) mutants recovered from 22 isolates of Sclerotinia homoeocarpa

\begin{tabular}{|c|c|c|c|c|c|c|c|}
\hline \multirow{2}{*}{ Isolate } & \multirow{2}{*}{$\begin{array}{l}\text { No. of colonies } \\
\left(\times 10^{5} \mathrm{cfu} / \mathrm{ml}\right)^{\mathrm{a}}\end{array}$} & \multirow{2}{*}{ No. of mutants ${ }^{b}$} & \multirow{2}{*}{$\begin{array}{l}\text { Mutation rate } \\
\qquad\left(\times 10^{5}\right)^{\mathrm{c}}\end{array}$} & \multicolumn{4}{|c|}{ nit mutant class ${ }^{\mathrm{d}}$} \\
\hline & & & & nit 1 & nit2 & nit3 & NitM \\
\hline A7 & $2.8 \pm 0.8$ & 0 & 0 & 0 & 0 & 0 & 0 \\
\hline ARK & $1.4 \pm 0.2$ & 0 & 0 & 0 & 0 & 0 & 0 \\
\hline BRS & $5.2 \pm 2.6$ & 0 & 0 & 0 & 0 & 0 & 0 \\
\hline FL17 & $4.9 \pm 0.8$ & 5 & $2.5 \pm 0.3$ & 4 & 0 & 0 & 1 \\
\hline I16 & $3.4 \pm 3.0$ & 4 & $4.8 \pm 4.2$ & 1 & 0 & 0 & 3 \\
\hline $\mathrm{I} 18$ & $3.0 \pm 0.7$ & 6 & $5.1 \pm 1.2$ & 4 & 0 & 0 & 2 \\
\hline L36 & $3.4 \pm 0.5$ & 9 & $7.5 \pm 10.6$ & 9 & 0 & 0 & 0 \\
\hline Les Bolstead & $4.4 \pm 0.8$ & 4 & $2.3 \pm 0.4$ & 1 & 0 & 0 & 3 \\
\hline MN1 & $3.3 \pm 0.7$ & 1 & $0.9 \pm 1.3$ & 1 & 0 & 0 & 0 \\
\hline Southbrook & $0.9 \pm 0.5$ & 1 & $5.0 \pm 7.1$ & 1 & 0 & 0 & 0 \\
\hline TB64D & $1.8 \pm 1.3$ & 3 & $5.0 \pm 1.8$ & 0 & 3 & 0 & 0 \\
\hline 30B-13 & $1.3 \pm 0.8$ & 5 & $11.3 \pm 4.2$ & 2 & 0 & 1 & 2 \\
\hline $30 \mathrm{~B}-24$ & $2.2 \pm 1.9$ & 0 & 0 & 0 & 0 & 0 & 0 \\
\hline $30 \mathrm{~B}-48$ & $2.7 \pm 1.2$ & 6 & $8.3 \pm 11.8$ & 6 & 0 & 0 & 0 \\
\hline $32-47$ & $2.6 \pm 0.4$ & 1 & $1.1 \pm 1.6$ & 1 & 0 & 0 & 0 \\
\hline $33 \mathrm{~A}-9$ & $1.4 \pm 0.1$ & 1 & $1.9 \pm 2.7$ & 1 & 0 & 0 & 0 \\
\hline $33 \mathrm{~A}-24$ & $2.1 \pm 0.6$ & 42 & $51.9 \pm 14.0$ & 22 & 0 & 0 & 20 \\
\hline $46-3$ & $1.7 \pm 0.9$ & 5 & $6.8 \pm 2.6$ & 3 & 0 & 1 & 1 \\
\hline $48-54$ & $6.4 \pm 0.5$ & 0 & 0 & 0 & 0 & 0 & 0 \\
\hline 64-14 & $1.6 \pm 0.6$ & 0 & 0 & 0 & 0 & 0 & 0 \\
\hline $64-41$ & $2.4 \pm 0.8$ & 3 & $3.6 \pm 2.7$ & 2 & 0 & 0 & 1 \\
\hline $64-49$ & $2.0 \pm 0.7$ & 5 & $7.0 \pm 4.2$ & 3 & 0 & 0 & 2 \\
\hline Total & & 101 & & 61 & 3 & 2 & 35 \\
\hline
\end{tabular}

${ }^{a} \mathrm{CFU}$ : colony-forming unit, number of colony produced from the shredded mycelial suspension on PDA (Potato Dextrose Agar).

${ }^{b}$ Number of nit mutants recovered from water agar media supplemented with $4.0 \%$ potassium chlorate.

${ }^{c}$ Mutation rate was determined as the number of nit mutants isolated on water agar medium amended with chlorate divided by the total number of CFUs on PDA per the same amount of mycelial suspension.

${ }^{\mathrm{d}}$ Nit mutant phenotypes determined according to growth on CDA amended with different nitrogen sources. nit 1 : mutation in a nitrate reductase structural locus, nit2: mutation in major nitrogen regulatory locus, nit3: mutation in a nitrate assimilation pathway-specific regulatory locus, NitM: mutation in one of five loci that affect the assembly of a molybdenum-containing cofactor necessary for nitrate reductase activity.

two additional attempts (Table 4). The nit mutants showed fast growth with thin and sparse colonies on WAC medium, and grew slowly on CDA containing only nitrate as the nitrogen source. The frequency of nit mutants significantly varied among the isolates tested. Over two runs of the experiment, isolate $33 \mathrm{~A}-24$ produced the greatest number of mutants: 42 mutants out of $2.1 \pm 0.6 \times 10^{5} \mathrm{cfu} / \mathrm{ml}$. In contrast, only one nit mutant was isolated from isolate MN1 out of $3.3 \pm 0.7 \times 10^{5} \mathrm{cfu} / \mathrm{ml}$ (Table 4 ).

Characterization of nit mutant phenotypes. At least one of the four nit mutant types were selected from 16 of the 22 isolates tested (Table 4). Of all mutants isolated, nit $1 \mathrm{mu}-$ tants were predominant (60.4\%) and NitM mutants were the second most prevalent (34.7\%). The frequency of nit 2 and nit 3 mutants was relatively rare at $3.0 \%$ and $2.0 \%$, respectively.

Vegetative compatibility assay. The complementation interaction of mutants exhibited abundant aerial mycelium growth at the junction zone where the two colonies met, and sometime extended to the entire colony (Fig. 1A). Two phenotypic types of incompatible reactions were observed (Figs. 1B-C). Strong incompatibility produced a distinct dark borderline where two colonies met 2-4 weeks after contact between two incompatible isolates (Fig. 1B). Weak incompatibility was characterized as formation of small spots of spontaneous aerial mycelium 2 weeks after contact but later a barrage with dark pigment was formed on the contacted zone after 30-days of incubation (Fig. 1C). 
Table 5. Complementation reactions among four types of nitrate nonutilizing (nit) mutants of Sclerotinia homoeocarpa

\begin{tabular}{ccccc}
\hline nit mutant phenotype $^{\mathrm{a}}$ & nit 1 & nit2 & nit3 & NitM \\
\hline nit 1 & - & + & - & + \\
nit2 & & - & + & + or $-{ }^{\mathrm{b}}$ \\
nit3 & & & - & + \\
NitM & & & & + or -
\end{tabular}

${ }^{\mathrm{a}}$ nit 1 : mutation in a nitrate reductase structural locus, nit2: mutation in major nitrogen regulatory locus, nit3: mutation in a nitrate assimilation pathway-specific regulatory locus, and NitM: mutation in one of five loci that affect the assembly of a molybdenum-containing cofactor necessary for nitrate reductase activity.

$\mathrm{b}_{-}=$prototrophic growth absent or inconspicuous between nit mutants of isolates. $+=$ dense prototrophic growth at the mycelial interface between nit mutants of isolates.

Under the compound microscope at 400X magnification, increased compartmentalization and vacuolization of hyphae were observed on the dark spots and borderline of the incompatible reaction. As a negative control, no reaction on CDA was produced between plugs of the same isolate (Figs. 1A-B) or single plug culture (Fig. 1D), whereas abundant aerial mycelium on PDA, a positive control medium (Fig. 1E).

Functional restoration after mutant complementation was more rapid and distinct in the pairings of NitM with nit 1 or nit 3 , and nit 2 with nit 1 or nit 3 combinations (Table 5). The complementary reactions between NitM and nit2 showed either abundant, profuse mycelium or no aerial mycelium. No clear complementation was observed on pairings between same mutant types of nit 1 , nit 2 and nit 3 , and between nit 1 and nit3. However, distinct complementation reactions of some pairing combinations between different NitM mutants produced rapid and robust heterokaryons with dense aerial mycelium. NitM or nit 2 mutants readily complemented with nit 1 and nit 3 mutants derived from the same VCG, producing distinct prototrophic aerial mycelium.

Out of the sixteen isolates produced nit mutants, 15 were amenable to the VCG assay and grouped into six VCGs based on pairings in all possible combinations between nit mutants (Table 1). Each VCG was designated as VCG 1 to VCG 6. VCGs 1 and 2 were dominant groups containing four isolates originating from different states. The VCGs 3 and 6 contained a single isolate.

Microsatellite marker analysis and concordance of nit mutant VCG and microsatellite genotypes. A total of 309 microsatellite loci were mined from genome sequence data: 148 di-nucleotide (48\%), 51 tri- (17\%), 47 tetra(16\%), 20 penta- (16\%), 26 hexa- $(8 \%), 8$ septa- $(3 \%)$,

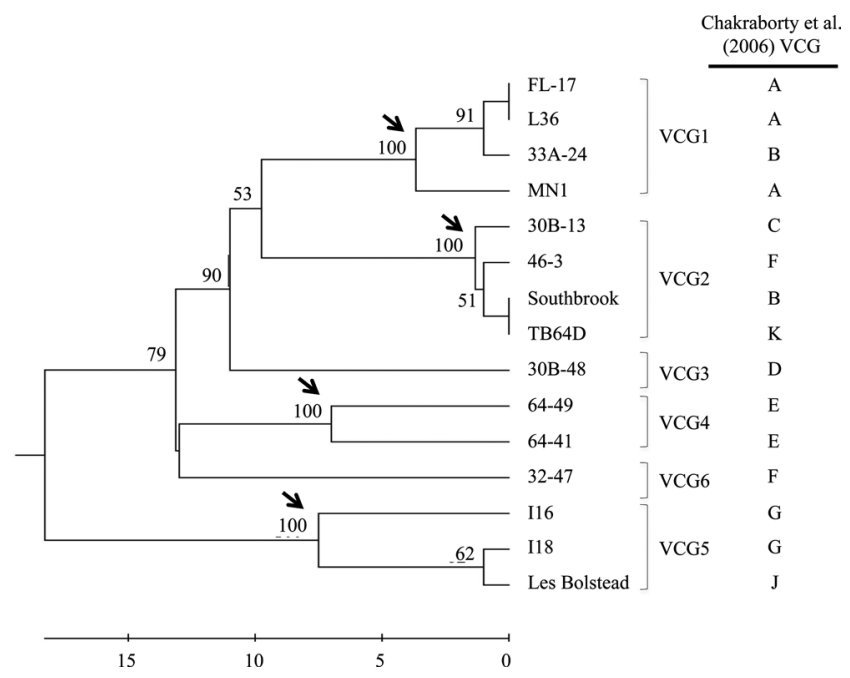

Fig. 2. UPGMA dendrogram of microsatellite genotypes of 15 isolates characterized using the improved nit mutant VCG assay. The nit mutant VCGs of taxa are indicated by brackets, and the VCGs of Chakraborty et al. (2006) (determined using the conventional method of Powell and Vargas, 2001) are indicated to the right of each taxon (Chakraborty et al., 2006; Powell and Vargas, 2001). Bootstrap proportions are indicated from 10,000 bootstrap replications. Arrows indicate bootstrap support for nit mutant VCGs containing multiple isolates.

and 9 octa- (3\%). Of these, 96 were screened for efficient amplification and single products. In total, 19 primer sets were selected, and the microsatellites produced a total of 57 alleles from the panel of all $22 \mathrm{~S}$. homoeocarpa isolates. Each marker produced from two to five alleles (Table 3). Sixteen of the microsatellite markers were amplified from the complete set of isolates, while SSR17616 wasn't amplified in isolates 64-49 and I16, SSR20589 wasn't amplified in isolate 32-47, and SSR22804 wasn't amplified in isolate 48-54. Distance analysis of the data set (including missing data) from the 15 isolates showed four groups with genetic similarity of $14 \%$ or less (Fig. 2, Table 6), with the exceptions of isolates 30B-48 of VCG3 and 32-47 from VCG6, which have isolated positions on the dendrogram.

The average genetic distances of isolates from within and among the VCGs with more than one isolate from three studies, including this one, are presented in Table 6. Genetic distances indicate that the six nit mutant VCGs found here, though they contained more isolates on average (2.67 \pm 1.21 ) than those of Chakraborty et al. (2006) included in the analysis $(1.6 \pm 0.70)$, displayed less average genetic distance $(7.72 \pm 4.93)$ than those $(13.4 \pm 4.7)$ of Chakraborty et al. (2006). Several VCGs of Chakraborty (2006) are nested within the nit mutant VCGs 1, 2, and 5 found in our current study (Table 6). 
Table 6. Summary of genetic distances from VCGs of the 14 isolates amenable to all three VCG assays: the current nit mutant VCG assay (1-6), the nit mutant VCG assay of Jo et al. (2008) (J1-J3), and the conventional VCG assay (A-K) (Chakraborty et al., 2006)

\begin{tabular}{cccc}
\hline VCG $^{\text {a }}$ & $\begin{array}{c}\text { Average \% } \\
\text { genetic distance } \\
\text { within VCG }\end{array}$ & $\begin{array}{c}\text { Number of } \\
\text { isolates }\end{array}$ & $\begin{array}{c}\text { Chakraborty et al. } \\
(2006) \text { VCGs nested } \\
\text { within }\end{array}$ \\
\hline 1 & 5.3 & 3 & A, B \\
2 & 2 & 4 & B, C, F, K \\
3 & - & 1 & D \\
4 & 14 & 2 & E \\
5 & 10.7 & 3 & G, J \\
6 & - & 1 & F \\
J1 & 18.7 & 10 & A, B, C, D, E, K \\
J2 & 10.7 & 3 & G, J \\
J3 & - & 1 & - \\
A & 5.3 & 3 & - \\
B & - & 1 & - \\
C & - & 1 & - \\
D & - & 1 & - \\
E & 14 & 2 & - \\
F & 21 & 2 & - \\
G & 15 & 2 & 1 \\
J & - & 1 & - \\
K & - & 1 & - \\
\hline
\end{tabular}

${ }^{\mathrm{a}}$ For the current nit mutant VCG assay (1-6), pairings among mutants of eighteen isolates recovered in Table 2 were made between two different nit mutants in all possible combinations [nit 1 (or nit3) and nit2 (or NitM)].

${ }^{\mathrm{b}}$ Average \% genetic distance was calculated by averaging all genetic distance values between isolates within VCG.
Table 8. Pairwise comparisons of microsatellite genotypes of isolates from four nit mutant VCGs found here using AMOVA. Probability values based on 9999 permutations are located above the diagonal, and pairwise $\phi_{\mathrm{PT}}$ values are located below the diagonal

\begin{tabular}{lllll}
\hline & VCG1 & VCG2 & VCG4 & VCG5 \\
\hline VCG1 & 0.000 & 0.028 & 0.066 & 0.029 \\
VCG2 & 0.838 & 0.000 & 0.069 & 0.029 \\
VCG4 & 0.768 & 0.760 & 0.000 & 0.092 \\
VCG5 & 0.810 & 0.851 & 0.689 & 0.000 \\
\hline
\end{tabular}

Analysis of molecular variance (AMOVA). The percentages of molecular variance within the nit mutant VCGs presented in our current study were $18.4 \%$, while among them the value was $81.6 \%$. The $\phi_{\mathrm{PT}}$ value, which is a measure of how much of the genetic variation is explained by the VCG groupings, was 0.795 , with $\mathrm{P}<0.001$. Conversely, among the two VCGs identified from Jo et al. which contained more than one isolate, the percentages of molecular variance were $81 \%$ within VCGs, and $19 \%$ among the two VCGs (Joaquim and Rowe, 1991). The $\phi_{\mathrm{PT}}$ value was 0.299, with $\mathrm{P}<0.006$. The percentages of molecular variance observed among and within the seven VCGs of Powell and Vargas (2001) with multiple isolates were 69.7\% and $30.3 \%$, respectively (Peakall and Smouse, 2006). The $\phi_{\mathrm{PT}}$ value was 0.572 , with $\mathrm{P}<0.001$ (Table 7) (Powell and Vargas, 2001). Pairwise $\phi_{\mathrm{PT}}$ values and probabilities from the AMOVA of the nit mutant VCGs are presented in Table 8.

Mitochondrial loci sequencing. Mitochondrial loci sequences including mtSSU, atp6-rns intergenic spacer re-

Table 7. Summary of AMOVA statistics for hierarchical analysis of Sclerotinia homoeocarpa isolates and the VCG designations of three separate studies

\begin{tabular}{|c|c|c|c|c|c|c|}
\hline Source of variation & & df & Sum of squares & $\begin{array}{c}\text { Percentage of } \\
\text { variation }\end{array}$ & $\phi_{\mathrm{PT}}$ & $P$ value \\
\hline \multirow[t]{2}{*}{ This study } & Among nit $\mathrm{VCG}$ & 3 & 120.68 & 81.6 & 0.795 & $<0.001$ \\
\hline & Within nit VCGs & 9 & 27.17 & 18.4 & 0.795 & $<0.001$ \\
\hline \multicolumn{2}{|l|}{ Total } & 12 & 147.85 & & & \\
\hline \multirow[t]{3}{*}{ Jo et al. (2008) } & Among nit $\mathrm{VCG}$ & 1 & 41.32 & 19.0 & 0.299 & $<0.006$ \\
\hline & Within nit VCGs & 16 & 180.68 & 81.0 & 0.299 & $<0.006$ \\
\hline & & 17 & 222.00 & & & \\
\hline \multirow[t]{2}{*}{ Chakraborty et al. (2006) } & Among nit $\mathrm{VCG}$ & 6 & 145.83 & 69.7 & 0.572 & $<0.001$ \\
\hline & Within nit VCGs & 12 & 63.33 & 30.3 & 0.572 & $<0.001$ \\
\hline Total & & 18 & 209.16 & & & \\
\hline
\end{tabular}


gion and COII were all identical among isolates representing each VCG.

\section{Discussion}

In the VCG assay based on nit-mutant complementation with four mutant types and over 100 mutants, 15 isolates of $S$. homoeocarpa were grouped into six VCGs (Table 1). These isolates were previously grouped into nine VCGs based on the macroscopic assessment of barrage formations and three VCGs based on nit-mutant complementation of Jo et al. (2008) which included three mutant types, less than half the number of mutants used here, and a much shorter incubation time for isolates being tested for complementation (10 days versus 30 days in this study) (Chakraborty et al., 2006; Jo et al., 2008; Powell and Vargas, 2001). The discordance of the nit mutant VCGs of Jo et al. (2008), and those presented here can be explained by the following caveats of the previous and updated nit mutant VCG assay types (Jo et al., 2008). While the conventional VCG assay relies on barrage formation only, the nit-mutant complementation assay relies on the ability of isolates to complement the nitrate assimilatory mutations of one another. Jo et al. (2008) scored several isolates as being vegetatively compatible, because they produced distinct or weak prototrophic mycelial growth indicative of complementation on nitrate medium with a lack of detectable barrage formation after 10 days of incubation (Jo et al., 2008). These isolates were placed preliminarily in the group 1 of Jo et al., the largest VCG that they found (Jo et al., 2008). This VCG is split into four VCGs using the updated nit mutant VCG assay in the current study, because after 30 days incubation, obvious and barrage zones were detected between the paired isolates and microscopic observations of incompatibility concurred with these results, indicating the heterokaryotic stage in weak spontaneous aerial mycelium is not stable.

The nit mutant VCGs we found were concordant with microsatellite genotype groupings in the UPGMA dendrogram of these 15 isolates, and each clade of isolates representing a VCG showed robust bootstrap support. In our knowledge, this is the first report showing genetic similarity of vegetative compatibility groupings in $S$. homoeocarpa. The VCGs of several fungi, including Verticillium, Cercospora, Aspergillus, and Botryosphaeria, have been shown concordant with genotype (Berbegal et al., 2010; Cai and Schneider, 2008; Cove, 1976; Grubisha and Cotty, 2009; Ma et al., 2004). In addition, the microsatellite groupings and nit mutant VCG groupings are also congruent with the genotype groupings of Chakraborty et al. (2006), which utilized RAPD markers to delineate the genotypic relationships among 23 isolates, 15 of which were characterized in our current study for nit mutant VCG (Chakraborty et al., 2006). Twenty-two of the 23 isolates of Chakraborty et al. (2006) were analyzed here with microsatellite genotyping and UPGMA analysis, and this tree gave the same overall topology (Chakraborty et al., 2006, unpublished data). Also, the AMOVA analysis results reported by Chakraborty et al. (2006) for the 23 isolates for which Powell and Vargas (2001) determined VCGs for were comparable to those presented in our study (Chakraborty et al., 2006). The convergence of groupings produced from our nit mutant VCGs and analysis of microsatellite genotypes from this study and RAPD genotype analysis of data of Chakraborty et al. (2006) respectively, suggests that isolates with similar genotypes are more likely to undergo nit-mutant complementation, and thus heterokaryon formation in the field (Chakraborty et al., 2006).

Within each of the VCGs B and F of Powell and Vargas (2001), there are isolates we characterized that are both not capable of nit complementation and are not genotypically similar in either of the RAPD or microsatellite analyses (Powell and Vargas, 2001). This raises concern that these isolates are likely not capable of heterokaryon formation, but yet they do not form a distinct barrage zone when they come in contact during the conventional VCG assay. Thus, the lack of a detectable incompatible interaction (barrage zone) was used to place them in the same VCG by Powell and Vargas (2001). However, our results suggest that the lack of a detectable barrage formation during the conventional VCG assay does not indicate the potential for heterokaryon formation between two $S$. homoeocarpa isolates. On the contrary, isolates from several of the VCGs of Powell and Vargas (2001) are nested within one of the six nit mutant VCGs presented here (Table 6). This suggests that these isolates appeared incompatible using the conventional VCG assay of Powell and Vargas (2001), and were assumed to be incapable of heterokaryon formation. However, we show that these isolates are indeed capable of heterokaryon formation and are genetically similar.

DeVries et al. (2008) and Viji et al. (2004) used amplified fragment length polymorphism (AFLP) genotyping to examine the genetic relatedness of conventionally determined VCGs and genotypic diversity, and they found similar results (DeVries et al., 2008; Warnke, 2003). In summary, both Viji et al. (2004) and DeVries et al. (2008) found representative isolates of VCGs A, B, C, $\mathrm{D}$ and $\mathrm{F}$ grouping with multiple other VCGs with high genetic similarity in the AFLP genotype analysis, and also 
that several isolates were found compatible to testers of multiple VCGs.

Even though this study was carried with a limited number of isolates, these results suggest that the conventional VCG assay may not be a reliable measure of the ability to form heterokaryons, and in keeping with previous studies, may not be a reliable indicator of genetic similarity (Chakraborty et al., 2006; DeVries et al., 2008; Warnke, 2003). However, there are several inherent difficulties in using the nit mutant system in place of the conventional VCG assay, since this system has recently introduced to $S$. homoeocarpa (Jo et al., 2008). Several isolates examined here were able to yield only a single mutant type per isolate, while others yielded more than two mutant types per isolate (Table 4). Isolate 33A-24 showed a ten-fold greater nit mutation rate than all other isolates screened, whereas six isolates failed to produce any mutants. Although the number of isolates tested was limited, these data suggest an isolate-dependent frequency of nit mutant generation, which is independent of genotype. Conversely, five of these six aforementioned isolates did yield nit mutants in the previous work. Also, even fewer nit3 mutants were selected here than previously reported with many fewer mutants by Jo et al. (2008). The inconsistencies in mutant generation for these isolates between this current study and the previous study may be due to deleterious effects brought on by repeated subculture of the isolates used. Continuous subculture in the present study has been shown to result in spontaneous genetic mutations affecting physiology and phenotype of a number of fungi, and perhaps this phenomenon may explain the differential formation of mutants among $S$. homoeocarpa isolates (Cox et al., 2007).

In the previous study of nit mutants in S. homoeocarpa by Jo et al. (2008) only three phenotypes (nit1, nit3, and NitM) of nit mutants were identified. In this current study, the nit2 mutant was also included in the VCG analysis, but was only produced in one isolate (Jo et al., 2008). The nit2 mutant type has also been previously recovered in Neurospora crassa (Marzluf, 1981) and Fusarium moniliforme (Klittich and Leslie, 1988). The dominant recovery of nit 1 and NitM mutants found in this study was consistent with the phenomenon previously reported in other plant-pathogenic fungi (Cecilia De Lima Favaro et al., 2007; Korolev and Katan, 1997; Nitzan et al., 2002). Obviously, the chance of selecting the rare nit2 mutant type increased as more mutants are isolated and screened. Given the high variability in mutant generation among isolates and mutant types within isolates (Table 4), the inherent difficulty in VCG determination using the conventional and nit-mutant methods can be complemented by microsatellite markers and other genotyping tools for measuring the variability of isolates from populations of $S$. homoeocarpa.

One putative clonal genotype was identified, and shared by two isolates FL-17 and L36 belonging to the same nit mutant VCG1, which were identical at 19 microsatellite loci despite the isolates FL17 and L36 were collected in Florida and Illinois, respectively. Viji et al. (2004) also document clonality of $S$. homoeocarpa isolates from geographically disparate locations, and found clonality among isolates collected nearly 30 years apart in Pennsylvania (Warnke, 2003). Given that $S$. homoeocarpa is thought to be an exclusively sterile fungus, low amounts of genetic diversity are to be expected, as has been suggested for $S$. homoeocarpa populations in New England (Mitkowski and Colucci, 2006). Lack of nucleotide substitutions in the mitochondrial loci sequenced from this panel of isolates from diverse locations support this hypothesis.

\section{Acknowledgments}

We thank K. Campbell-Nelson and J. Popko for editorial review of the manuscript.

\section{References}

Altschul, S. F., Madden, T. L., Schaffer, A. A., Zhang, J., Zhang, Z., Miller, W. and Lipman, D. J. 1997. Gapped BLAST and PSI-BLAST: a new generation of protein database search programs. Nucleic Acids Res. 25:3389-3402.

Baldwin, N. A. and Newell, A. J. 1992. Field production of fertile apothecia by Sclerotinia homoeocarpa in Festuca turf. $J$. Sports Turf Res. Inst. 68:73-76.

Bennett, F. T. 1937. Dollar spot disease on turf and its causal organism Sclerotinia homoeocarpa n. sp. Ann. Appl. Biol. 24:236-257.

Berbegal, M., Ortega, A., Jiménez-Gasco, M. M., OlivaresGarcía, C., Jiménez-Díaz, R. M. and Armengol, J. 2010. Genetic diversity and host range of Verticillium dahliae isolates from artichoke and other vegetable crops in Spain. Phytopathology 94:396-404.

Brooker, N. L., Leslie, J. F. and Dickman, M. B. 1991. Nitrate nonutilizing mutants of Colletotrichum and their use in studies of vegetative compatibility and genetic relatedness. Phytopathology 81:672-677.

Burpee, L. L. 1997. Control of dollar spot of creeping bentgrass caused by an isolate of Sclerotinia homoeocarpa resistant to benzimidazole and demethylation-inhibitor fungicides. Plant Dis. 81:1259-1263.

Cai, G. and Schneider, R. W. 2008. Population structure of Cercospora kikuchii, the causal agent of Cercospora leaf blight and purple seed stain in soybean. Phytopathology 98:823-829. 
Cecilia De Lima Favaro, L., Luiz Araujo, W., Aparecida De Souza-Paccola, E., Lucio Azevedo, J. and Paccola-Meirelles, L. D. 2007. Colletotrichum sublineolum genetic instability assessed by mutants resistant to chlorate. Mycol. Res. 111:93105.

Chakraborty, N., Chang, T., Casler, M. D. and Jung, G. 2006. Response of bentgrass cultivars to Sclerotinia homoeocarpa isolates representing 10 vegetative compatibility groups. Crop Sci. 46:1237-1244.

Chang, S. W., Chang, T. H., Hong, J. K., Park, J. H. and Jung, S. W. 2011. Vegetative compatibility grouping of Sclerotinia homoeocarpa isolates infecting turfgrass in South Korea. Asian J. Turfgrass Sci. 25:171-176.

Chang, S. W., Jung, S. W., Kim, S., Park, J. H. and Lee, J. Y. 2012. Synergistic interaction of fungicides in mixtures under different conditions of dollar spot disease caused by Sclerotinia homoeocarpa. Asian J. Turfgrass Sci. 26:96-101. (in Korean).

Chang, S. W., Jung, S. W., Kim, S., Park, J. H. and Lee, J. Y. 2013. Control effect on dollar spot disease caused by Sclerotinia homoeocarpa under different application rates and intervals with two mixed fungicides. Weed \& Turf. Sci. 2:408-412. (in Korean).

Correll, J. C., Klittich, C. J. R. and Leslie, F. F. 1987. Nitrate nonutilizing mutants of Fusarium oxysporum and their use in vegetative compatibility tests. Phytopathology 77:16401646.

Correll, J. C., Gordon, T. R. and McCain, A. H. 1988. Vegetative compatibility and pathogenicity of Verticillium albo-atrum. Phytopathology 78:1017-1021.

Cove, D. J. 1976. Chlorate toxicity in Aspergillus nidulansselection and characterization of chlorate resistant mutants. Heredity 36:191-203.

Cox, K. D., Bryson, P. K. and Schnabel, G. 2007. Instability of propiconazole resistance and fitness in Monilinia fructicola. Phytopathology 97:448-453.

DeVries, R. E., Trigiano, R. N., Windham, M. T., Windham, A. S., Sorochan, J. C., Rinehart, T. A. and Vargas, J. M. 2008. Genetic analysis of fungicide-resistant Sclerotinia homoeocarpa isolates from Tennessee and Northern Mississippi. Plant Dis. 92:83-90.

Felsentein, N. J. 1989. PHYLIP-phylogeny inference package (version 3.2). Cladistics 5:164-166.

Ghikas, D. V., Kouvelis, V. N. and Typas, M. A. 2010. Phylogenetic and biogeographic Implications inferred by mitochondrial intergenic region analyses and ITS1-5.8S-ITS2 of the entomopathogenic fungi Beauveria bassiana and B. brongniartii. BMC Microbiol. 10:1-15.

Grubisha, L. C. and Cotty, P. J. 2009. Twenty-four microsatellite markers for the aflatoxin-producing fungus Aspergillus flavus. Mol. Ecol. Res. 9:264-267.

Glass, N. L., Jacobson, D. J. and Shiu, P. K. T. 2000. The genetics of hyphal fusion and vegetative incompatibility in filamentous Ascomycete fungi. Annu. Rev. Genet. 34:165-186.
Glass, N. L., Rasmussen, C., Roca, M. G. and Read, N. D. 2004. Hyphal homing, fusion and mycelial interconnectedness. TRENDS in Microbiol. 12:135-141.

Jackson, N. 1973. Apothecial production in Sclerotinia homoeocarpa F. T. Bennett. J. Sports Turf Res. Inst. 49:5863.

Joaquim, T. R. and Rowe, R. C. 1991. Vegetative compatibility and virulence of strains of Verticillium dahliae from soil and potato plants. Phytopathology 81:552-558.

Jo, Y. K., Chang, S. W., Rees, J. and Jung, G. 2008. Reassessment of vegetative compatibility of Sclerotinia homoeocarpa using nitrate-nonutilizing mutants. Phytopathology 98:108-114.

Katan, T. and Katan, J. 1988. Vegetative compatibility grouping of Fusarium oxysporum f. sp. vasinfectum from tissue and rhizosphere of cotton plants. Phytopathology 78:852-855.

Klittich, C. J. R. and Leslie, J. F. 1988. Nitrate reduction mutants of Fusarium moniliforme (Gibberella fujikuroi). Genetics 118:417-423.

Korolev, N. and Katan, T. 1997. Improved medium for selecting nitrate non-utilizing (nit) mutants of Verticillium dahlia. Phytopathology 87:1067-1070.

Lamour, K. H., Finley, L., Hurtado-Gonzalez, O., Gobena, D., Tierney, M. and Meijer, H. J. G. 2006. Targeted gene mutation in Phytophthora sp. Mol. Plant Microbe In. 19:1359-1367.

Leslie, F. J. 1993. Fungal vegetative compatibility. Annu. Rev. Phytopathol. 31:127-150.

Ma, Z., Luo, Y. and Michailides, T. J. 2004. Spatiotemporal changes in the population structure of Botryosphaeria dothidea from California pistachio orchards. Phytopathology 94:326-332.

Marlatt, M. L., Correll, J. C., Kaufmann, P. and Cooper, P. E. 1996. Two genetically distinct populations of Fusarium oxysporum f. sp. lycopersici race 3 in the United States. Plant Dis. 80:1336-1342.

Marzluf, G. A. 1981. Regulation of nitrogen metabolism and gene expression in fungi. Microbiol. Rev. 45:437-461.

Mitkowski, N. A. and Colucci, S. 2006. The identification of a limited number of vegetative compatibility groups within isolates of Sclerotinia homoeocarpa infecting Poa spp. and Agrostis palustris from temperate climates. J. Phytopathology 154:500-503.

Nitzan, N., Hazanovsky, M., Tal, M. and Tsror (Lahkim), L. 2002. Vegetative compatibility groups in Colletotrichum coccodes, the causal agent of black dot on potato. Phytopathology 92:827-832.

Peakall, R. and Smouse, P. E. 2006. GenAlEx 6: genetic analysis in Excel. Population genetic software for teaching and research. Mole. Ecol. Notes 6:288-295.

Powell, J. F. and Vargas, J. M. 2001. Vegetative compatibility and seasonal variation among isolates of Sclerotinia homoeocarpa. Plant Dis. 85:377-381.

Rozen, S. and Skaletsky, H. 2000. Primer 3 on the WWW for general users and biologist programmers. In Bioinformatics Methods and Protocols: Methods in Molecular Biology (eds 
Krawetz S, Misener S), pp. 365-386. Humana Press, Totowa, New Jersey.

Saitoh, K., Togashi, K., Arie, T. and Teraoka, T. 2006. A simple method for a mini-preparation of fungal DNA. J. Gen. Plant. Pathol. 72: 348-350.

Skovgaard, K., Nirenberg, H. I., O’Donnell, K. and Rosendahl, S. 2001. Evolution of Fusarium oxysporum f. sp. vasinfectum races Inferred from multigene genealogies. Phytopathology 91:1231-1237.

Smiley, R. W. Dernoeden, P. H. and Clarke B. B. 2005. Compendium of turfgrass diseases. American Phytopathological Society, St. Paul, MN.

Smith, J. D., Jackson, N. and Woolhouse, A. R. 1989. Fungal diseases of amenity turfgrasses. 3rd. Ed. E. and F. Spon, London.

Subbarao, K. V., Chassot, A., Gordon, T. R., Hubbard, J. C., Bonello, P., Mullin, R., Okamoto, D., Davis, R. M. and Koike,
S. T. 1995. Genetic relationships and cross pathogenicities of Verticillium dahliae isolates from cauliflower and other crops. Phytopathology 85:1105-1112.

Tamura, K., Dudley, J., Nei, M. and Kumar, S. 2007. MEGA4: Molecular evolutionary genetics analysis (MEGA) software version 4.0. Mol. Biol. Evol. 24:1596-1599.

Tomsett, A. B. and Garrett, R. H. 1980. The isolation and characterization of mutants defective in nitrate assimilation in $\mathrm{Neu}$ rospora crassa. Genetics 95:649-660.

Viji, G., Uddin, W., O’Neill, N. R., Mischke, S. and Saunders, J. A. 2004. Genetic diversity of Sclerotinia homoeocarpa isolates from turfgrasses from various regions in North America. Plant Dis. 88:1269-1276.

Warnke, S. 2003. Creeping bentgrass (Agrostis stolonifera L.). Pages 175-185 in: Turfgrass Biology, Genetics, and Breeding. M. D. Casler and R. R. Duncan, eds. John Wiley \& Sons, Hoboken, NJ. 